\title{
ON A QUESTION CONCERNING PARTITIONING RAISED BY R. H. BING
}

\section{LIDA K. BARRETT}

\section{R. H. Bing on p. 341 of [1] raises the following question:}

Question. Does there exist a positive integer $n$ such that the following result holds for each continuous curve $M$, each positive number $\epsilon$, and each pair of mutually exclusive closed subsets $H$ and $K$ of $M$ ? If $R$ is a finite subset of $M$ such that each point of $R$ belongs to an arc in $M$ of diameter less than $\epsilon$ that intersects $H+K$, there are two collections $A_{H}$ and $A_{K}$ of arcs satisfying the following conditions: (a) Each element of $A_{H}$ intersects $H$ but not $K$ and each element of $A_{K}$ intersects $K$ but not $H$ nor any element of $A_{H}$. (b) Each element of $R$ belongs to an element of $A_{H}+A_{K}$. (c) Each element of $A_{H}+A_{K}$ is of diameter less than $n \epsilon$.

If for some integer $n$ the answer is yes, then E. E. Moise's method of partitioning would be validated (see [2] and [3]). Also, a simple technique yielding an affirmative answer would allow a more direct proof of partitioning by Bing's method and could probably be used to advantage on other problems.

Bing [1] gives an example to show that the answer is no for $n=1$. The present paper gives an example to show that the answer is no for $n=2$.

In the example given below, a metric will be defined such that with this metric the given point set has the desired metric property. The example was originally considered with a homeomorphism to Euclidean three space, where the image had the desired property. The metric given here follows a suggestion of R. H. Bing.

ExAmple. The example is described at the top of the following page.

The metric. Consider the example as a finite graph $G$, the sum of a finite number of segments, $s_{1}, s_{2}, \cdots, s_{n}$, such that if $s_{i}$ and $s_{j}$ have a point in common, $s_{i} \cdot s_{j}$ is an end point of both $s_{i}$ and $s_{j}$, and such that each element $a_{i}$ is the sum of elements of $s_{1}, s_{2}, s_{3}, \cdots, s_{n}$. Let each element $s_{i}$ be of length slightly less than $\epsilon$, say $\epsilon-\delta$. Now if $v_{i}, v_{j}$ are vertices of segments of $G$, set $d\left(v_{i}, v_{j}\right)=0$ if $i=j, d\left(v_{i}, v_{j}\right)$ $=N(\epsilon-\delta)$ if $i \neq j$, where $N$ is the minimum number of $a_{i}$ 's whose sum is a continuum containing $v_{i}$ and $v_{j}$. If $x, y$ belongs to the same $a_{i}$, let $d(x, y)=\min$ (distance $x$ to $y$ along the line segment, $\epsilon-\delta$ ).

Presented to the Society, December 27, 1955; received by the editors April 28, 1956 and, in revised form, May 26, 1956 and September 22, 1956. 


\section{EXAMPLE}

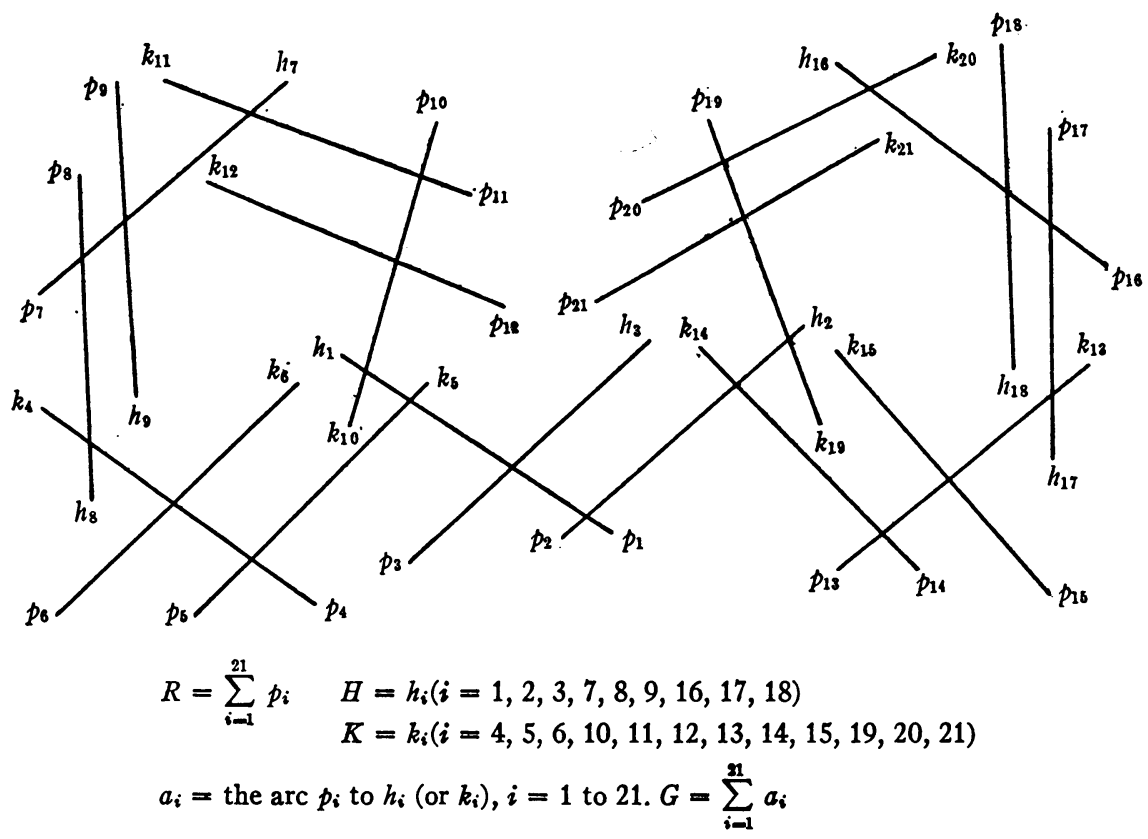

Then for arbitrary $x, y$ in $G$ set $d(x, y)=\min (d(x, p)+d(p, q)$ $+d(q, y))$ where minimum is taken over all $p, q$ where $d(p, q)$ has already been defined.

Indication of a proof. Assume that $n$ is two. Consider $\operatorname{arcs} a_{1}, a_{2}, a_{3}$ (from $p_{1}$ to $h_{1}, p_{2}$ to $h_{2}$, and $p_{3}$ to $h_{3}$ ). Note that since $n=2, p_{3}$ must belong to an arc in $A_{H}$ that is a subset of $a_{3}$ or $a_{3}+a_{1}$. Then $p_{1}$ belongs to an arc of $A_{H}$ as does $p_{2}$. This means that either:

Case I. $a_{1}$ belongs to $A_{H^{*}}$; or,

Case II. $a_{2}$ belongs to $A_{H^{*}}$.

Suppose Case I. Then $a_{4}$ must belong to $A_{K^{*}}, a_{7}$ to $A_{H^{*}}$, and $a_{10}$ to $A_{K^{*}}$, but $a_{10}$ intersects $a_{1}$, which gives a contradiction. A similar line of reasoning leads to a contradiction for Case II.

\section{REFERENCES}

1. R. H. Bing, Partitioning continuous curves, Bull. Amer. Math. Soc. vol. 58 (1952) pp. 536-556.

2. E. E. Moise, Grille decomposition and convexification theorems for compact metric continua, Bull. Amer. Math. Soc. vol. 55 (1949) pp. 1111-1121.

3. - A note of correction, Proc. Amer. Math. Soc. vol. 2 (1951) p. 838.

University of ConNecticut (Waterbury Branch) 